\title{
Review: brief primary care interventions are moderately effective for increasing physical activity
}

Eakin EG, Glasgow RE, Riley KM. Review of primary care-based physical activity intervention studies. Effectiveness and implications for practice and future research.J Fam Pract 2000 Feb;49:158-68.

\author{
QUESTION: In family practice settings, how effective are interventions aimed at \\ increasing physical activity?
}

\section{Data sources}

Studies were identified by searching Medline (1980-98), Psychological Abstracts, ERIC, and Healthstar databases and the Journal of Family Practice web site with the terms physical activity, physical activity counselling, primary care, medical office, exercise interventions, and health promotion. Bibliographies of relevant studies and previous reviews were scanned, and 3 experts in the field were contacted to identify unpublished studies.

\section{Study selection}

Studies were selected if they were randomised controlled trials (RCTs) or quasi-experimental studies, the intervention was delivered or initiated in a primary care setting, and the results included $\geqslant 1$ measure of physical activity. Studies focusing solely on cardiovascular disease were excluded.

\section{Data extraction}

Data on methodological quality were extracted by 2 independent reviewers based on the RE-AIM framework for evaluating public health interventions. RE-AIM assesses 5 dimensions: Reach, Efficacy, Adoption, Implementation, and Maintenance. Methodological criteria for this review were study design, analyses, dependent variable, reach, implementation, and attrition (maximum score of 10 points). Data were also extracted on sample size, physical activity intervention, follow up, and short $(<12 \mathrm{mo})$ and long term $(\geqslant 12 \mathrm{mo})$ outcomes.

\section{Main results}

Of 15 included studies, 10 were RCTs involving 15208 patients (age range 18-80 y). The methods scores of the RCTs ranged from 4 to 9 . Physical activity interventions included $\geqslant 1$ of physician advice or counselling ( 2 to 60 min); a written physical activity prescription; an instructional handout or video; a follow up visit with a clinician or health educator; an exercise programme at a community centre; or a 2 hour workshop. All studies used patient self reports of physical activity. 7 studies reported short term outcomes (range 4 wks to $8 \mathrm{mo}$ ), and 4 studies reported long term outcomes (range 1 to 4 y) (1 study reported both short and long term outcomes). Of the 7 studies reporting short term outcomes, 2 showed no difference, and 5 showed an increase in physical activity or exercise in the treatment groups, including 1 that showed an increase in duration of physical activity but not frequency. Of the 4 studies with long term outcomes, 2 showed a benefit: increased stretching and metabolic rate (effect sizes 0.14 and 0.09 , respectively, $\mathrm{p}<0.001$ for both) in 1 study and a decrease in sedentary lifestyle (odds ratio 1.28, $\mathrm{p}<0.05$ ) in the other.

\section{Conclusion}

Primary care based interventions are moderately effective in increasing physical activity in the short term; some interventions may also be effective in the long term.

\section{COMMENTARY}

Regular exercise prevents disease and promotes health. This is well accepted by patients, providers, and public health experts. The US Preventive Services Task Force has consistently recommended counselling to promote physical activity for all adults. ${ }^{1}$ Until recently, however, little evidence existed that such counselling made a difference. Eakin $e t$ al systematically reviewed the literature on the effectiveness of exercise counselling in primary care. They concluded that brief primary care based physical activity counselling is modestly effective, particularly in the short term. They suggested (1) tailoring counselling to the patient and providing written materials; (2) focusing on physical activity initially rather than on multiple prevention recommendations; and (3) using other trained members of the healthcare team to counsel patients.

If you are a busy clinician who is convinced of the benefits of exercise and interested in counselling your patients, what should you do? The best answer is not to try harder. The literature indicates that you should redesign your system to better support screening and counselling.' For example, patients might answer questions on physical activity before seeing the provider, either by self administered questionnaire or by report when vital signs are assessed. An exercise pamphlet could be placed in appropriate charts, reminding you to counsel and giving you something to tailor. Office staff could handle additional counselling and clarification. Perhaps most important, measuring and monitoring counselling rates will help to ensure that most patients are counselled about exercise. This review confirms our previously held belief that such counselling does indeed make a difference.

Stephanie P Fein, MD Scott E Sherman, MD, MPH VA Center for the Study of Healthcare Provider Behavior Los Angeles, California, USA

1 US Preventive Services Task Force. Guide to clinical preventive services. 2d edition. Rockville, MD: Agency for Healthcare Research and Quality; 1996:611-24. http://www.ahcpr.gov/ clinic/cpsix.htm.

2 Solberg LI, Kottke TE, Conn SA, et al. Delivering clinical preventive services is a systems problem. Ann Behav Med 1997;19:271-8. 